\title{
A Simple Assumed Strain Method for Enhancing the Accuracy of the Cubic Triangular $C^{0}$ Plate Bending Element
}

\author{
K.Y.Sze and D.Zhu* \\ Department of Mechanical Engineering, The University of Hong Kong \\ Pokfulam Road, HONG KONG
}

\begin{abstract}
SUMMARY
The conventional cubic triangular Mindlin/Reissner plate bending element, DISP10, is in general too stiff. To reduce the element stiffness, three of the strain sampling points are shifted from the integration stations to the element corners so as to reduce the number of shear constraints in the global level. The strain field is then obtained by interpolation. In this way, the constraint ratio of the element increases from 1.125 to 1.5 which is exactly equal to a postulated optimal value. However, the element does not appear to be more accurate than its conventional counterpart. While keeping the constraint ratio and interpolation pivots unchanged, two different ways of refining the sampled strains at the element corners are attempted and the pertinent elements are consistently more accurate than DISP10.
\end{abstract}

Keywords: finite element, plate, triangular, assumed strain, ten-node, cubic

* on leave from : Southwest Jiaotong University, Chengdu 610013, P.R.CHINA 


\section{INTRODUCTION}

As motivated by the need of advanced triangular elements for automatic mesh generation and refinement, quadratic or six node Mindlin/Reissner or $\mathrm{C}^{0}$ plate bending elements have been developed in our previous papers [1,2]. These elements are developed by observing the following criteria : (a) their kinematics has not been modified by the Kirchhoff constraints, (b) they possess no commutable mechanism, (c) they do not exhibit shear locking, (d) they pass the constant moment and constant transverse shear patch tests, (e) their boundary nodes have the same number of d.o.f.s so that there would be no complication for being implemented in all finite element codes. Moreover, two quadratic curved shell elements have also been developed by including the requirements of (f) satisfying the 2/D membrane patch test and (g) being free from membrane locking $[3,4]$.

Same as the conventional quadratic triangular (six-node) Mindlin/Reissner plate bending element, the cubic (ten-node) one, abbreviated as DISP10, is in general too stiff. It is also noted that there are only very few articles devoted to the improvement of DISP10. Among them, Lee, Dai \& Yeom proposed two ten-node hybrid strain elements which contain six and twelve assumed shear strain modes [5]. In this paper, shear refers to transverse shear unless otherwise specified. The former hybrid element does not suffer from shear locking but exhibits spurious zero energy modes. On the other hand, the latter suffers from shear locking but does not exhibit any spurious zero energy modes. A quadrilateral macro-element composed of two triangular elements of each kind was formed, see Fig.1a. The macro-element does not suffer from shear locking and spurious zero energy modes. Unfortunately, it no more possesses the advantage of triangular elements in automatic mesh generation and refinement. Another cubic triangular element is MITC12 which is a assumed strain element [6,7]. It contains nine boundary nodes and four bubble nodes. Three of the bubble nodes are equipped with rotational d.o.f.s only whereas the remaining one is equipped with translational d.o.f.s only, see Fig.1b. The shear strain field is constructed from fifteen shear strain samples out of which nine are tangential to the element edges. To our best knowledge, MITC12 is the only cubic (or close to cubic) element that fulfills criteria (a) to (e). Nevertheless, MITC12 is rather expensive due to the presence of the four bubble nodes and the required thirteen-point numerical integration scheme.

To reduce the element stiffness, three of the strain sampling points are shifted from the integration stations to the element corners. The constraint ratio of the element is then increased from 1.125 to 1.5. The latter is the optimal value suggested in the textbook of Hughes [8]. However, the resulting element does not appear to be more accurate than DISP10. As an attempt to improve the accuracy, the shear strains at the element corners are obtained by interpolating the natural shear 
strain along the element edges. Two sets of interpolation pivots are employed. It can be seen from the numerical benchmark tests that the attempt leads to marked improvement of accuracy.

\section{CONVENTIONAL ELEMENT}

A ten-node triangular element is shown in Fig.2a. The area coordinates $(r, s, t)$ are employed as the parametric coordinates and

$$
r+s+t=1
$$

The mid-plane of the element coincides with the $z=0$ plane. The element geometry and kinematics are interpolated as :

$$
x=\sum_{i=1}^{10} N_{i} x_{i}, y=\sum_{i=1}^{10} N_{i} y_{i},\left\{\begin{array}{c}
w \\
\theta_{x} \\
\theta_{y}
\end{array}\right\}=\sum_{i=1}^{10} N_{i}\left\{\begin{array}{c}
w_{i} \\
\theta_{x i} \\
\theta_{y i}
\end{array}\right\}=\mathbf{N} \mathbf{q}
$$

where

$$
\begin{aligned}
& N_{1}=(3 r-1)(3 r-2) r / 2, N_{2}=9 r s(3 r-1) / 2, N_{3}=9 r s(3 s-1) / 2 \\
& N_{4}=(3 s-1)(3 s-2) s / 2, N_{5}=9 \operatorname{st}(3 s-1) / 2, N_{6}=9 \operatorname{st}(3 t-1) / 2 \\
& N_{7}=(3 t-1)(3 t-2) t / 2, N_{8}=9 \operatorname{tr}(3 t-1) / 2, N_{9}=9 \operatorname{tr}(3 r-1) / 2, N_{10}=27 r s t \\
& \left(x_{i}, y_{i}\right) \text { is the nodal coordinates of the i-th node } \\
& \mathbf{N}=\left[N_{1} \mathbf{I}_{3}, \ldots, N_{10} \mathbf{I}_{3}\right] \text { is the interpolation matrix, } \mathbf{I}_{3} \text { is the third order identity matrix } \\
& \mathbf{q}=\left[w_{1}, \theta_{x 1}, \theta_{y 1}, \ldots, w_{10}, \theta_{x 10}, \theta_{y 10}\right]^{T} \text { is the element displacement vector }
\end{aligned}
$$

The displacement components at any point $(x, y, z)$ inside the element are governed by :

$$
u=u(x, y, z)=z \theta_{y}(x, y), v=v(x, y, z)=-z \theta_{x}(x, y), w=w(x, y)
$$

The in-plane and out-of-plane strain components are derived by invoking Eqn.(2) and Eqn.(3) :

$$
\boldsymbol{\varepsilon}=\left\{\begin{array}{c}
\varepsilon_{x} \\
\varepsilon_{y} \\
\gamma_{x y}
\end{array}\right\}=\left\{\begin{array}{c}
\partial_{x} u \\
\partial_{y} v \\
\partial_{y} u+\partial_{x} v
\end{array}\right\}=z\left\{\begin{array}{c}
\partial_{x} \theta_{y} \\
-\partial_{y} \theta_{x} \\
-\partial_{x} \theta_{x}+\partial_{y} \theta_{y}
\end{array}\right\}=z \mathbf{B}_{b} \mathbf{q}
$$




$$
\gamma=\left\{\begin{array}{l}
\gamma_{z x} \\
\gamma_{z y}
\end{array}\right\}=\left\{\begin{array}{l}
\partial_{x} w+\partial_{z} u \\
\partial_{y} w+\partial_{z} v
\end{array}\right\}=\left\{\begin{array}{l}
\partial_{x} w+\theta_{y} \\
\partial_{y} w-\theta_{x}
\end{array}\right\}=\mathbf{B}_{\gamma} \mathbf{q}, \varepsilon_{z}=\partial_{z} w=0
$$

where

$$
\mathbf{B}_{b}=\left[\begin{array}{ccc}
0 & 0 & \partial_{x} \\
0 & -\partial_{y} & 0 \\
0 & -\partial_{x} & \partial_{y}
\end{array}\right] \mathbf{N}, \mathbf{B}_{\gamma}=\left[\begin{array}{ccc}
\partial_{x} & 0 & 1 \\
\partial_{y} & -1 & 0
\end{array}\right] \mathbf{N}, \partial_{x}=\frac{\partial}{\partial x} \text { etc. }
$$

After integrating the strain energy from $z=-h / 2$ to $z=+h / 2$ where $h$ is the plate thickness, the stiffness matrix of the DISP10 is obtained :

$$
\mathbf{k}_{D I S P 10}=\int_{0}^{1} \int_{0}^{1-t}\left(\frac{h^{3}}{12} \mathbf{B}_{b}^{T} \mathbf{C}_{\varepsilon} \mathbf{B}_{b}+h \mathbf{B}_{s}^{T} \mathbf{C}_{\gamma} \mathbf{B}_{s}\right) J d s d t
$$

where

$$
J=\operatorname{det} .\left[\begin{array}{cc}
\partial_{s} x & \partial_{s} y \\
\partial_{t} x & \partial_{t} y
\end{array}\right]
$$

Moreover, $\mathbf{C}_{\varepsilon}$ and $\mathbf{C}_{\gamma}$ are the material stiffness matrices for the inplane (plane stress) and transverse shear responses, respectively. For an isotropic material with elastic modules $E$ and Poisson's ratio $v$,

$$
\mathbf{C}_{b}=\frac{E}{1-v^{2}}\left[\begin{array}{ccc}
1 & v & 0 \\
v & 1 & 0 \\
0 & 0 & (1-v) / 2
\end{array}\right], \mathbf{C}_{s}=\frac{k E}{2(1+v)}\left[\begin{array}{ll}
1 & 0 \\
0 & 1
\end{array}\right]
$$

where $k$ is shear correction factor commonly taken to be $5 / 6$. The six-point integration rule is sufficient to ensure the proper element rank, see Fig.2a. The $(r, s, t)$ values of the integration stations $a, b$ and $c$ are permutations of $\alpha, \alpha$ and $1-2 \alpha$ where $\alpha \approx 0.445948$. On the other hand, $(r, s, t)$ values of the integration stations $d, e$ and $f$ are permutations of $\beta, \beta$ and $1-2 \beta$ where $\beta \approx 0.091576$ [9].

\section{CONSTRAINT COUNTING AND AST10 ELEMENTS}


As shear deformation is taken into account by $\mathrm{C}^{0}$ elements, they are more suitable than $\mathrm{C}^{1}$ elements for analysing thick, composite and sandwich plates in which shear deformation cannot be ignored. However, $\mathrm{C}^{0}$ elements suffer from shear locking when the shear energy is not properly monitored. The numerical penalty associated with the shear energy is important for enforcing the Kirchhoff or (zero) shear constraints. However, if there are too many constraints in an element assembly, the latter can hardly be deflected and behaves as if locked. A simple method widely used by element designers for pre-identifying locking is the constraint count [8-10]. When a simple quadrilateral element patch is assembled to an existing mesh, it brings forward a number of $N K$ independent kinematic d.o.f.s and a number of $N C$ independent Kirchhoff constraints. Constraint ratio, $C R$, is the ratio of $N K$ to $N C$. If $C R$ is too small, the element will be too stiff and may even exhibit shear locking. If $C R$ is too large, the Kirchhoff or thin plate limit is poorly approximated and the element will be too flexible. In the textbook of Hughes, the optimal $C R$ value for $C^{0}$ plate bending problems is postulated to be $3 / 2$ [8].

Fig.3a shows the constraint count of DISP10. Along the hatched edges, all d.o.f.s are preexisting. Thus, $N K$ equals 27. As two shear strains are sampled at each of the integration points and there are six integration points per element, $N C=24$ and $C R=1.125$ which suggests that the element is too stiff. Realizing that the edge shear strain which abbreviates the tangential transverse shear strain at an element edge involves the kinematic d.o.f.s associated with the pertinent element edge only, the edge shear strains sampled at the same point by two adjacent elements are identical. Hence, the edge shears at the hatched edges do not contribute to $N C$ and that at the common element edges are dependent. With this in mind, $N C$ for MITC12, see Fig. 3b, should be 21 and thus $C R=35 / 21=1.667$ which may be too flexible but is still quite close to the optimal value.

A possible way of reducing $N C$ of DISP10 is to sample some of the shear strains at the element boundary. One way of doing this is to replace integration stations $d, e$ and $f$, see Fig.2a, by the three element corners. A quadratic shear strain field is then interpolated by using the element corners and integration stations $a, b$ and $c$ as the pivots, see Fig.3c. This assumed strain element will be termed AST10C where "C" designates corner. Noting that the strains derived at the element corners can be re-constituted in such a way that both of them become edge strains, $N C$ and $C R$ for the setup are 18 and 1.5 , respectively.

For the purpose of forming the element stiffness matrix of AST10C, only the strains at the integration stations are required. Consider the interpolated $\gamma_{z x}$ field, it can be expressed as :

$$
\tilde{\gamma}_{z x}=\mathbf{F}(s, t)\left[\gamma_{z x a}, \gamma_{z x b}, \gamma_{z x c}, \tilde{\gamma}_{z x d}, \tilde{\gamma}_{z x e}, \tilde{\gamma}_{z x f}\right]^{T}
$$


where $\mathbf{F}(s, t)$ is the $1 \times 6$ interpolation matrix, $a, b, c \ldots$ refer to the corresponding integration stations and the over-tiles indicate that the strains are obtained by interpolation instead of applying the strain-displacement operators to the interpolated displacement/rotations. By substituting the $s-t$ coordinates of node 1, node 4, node 7 and the integration stations $a, b, c$ into Eqn.(7), we have

$$
\left\{\begin{array}{l}
\gamma_{z x a} \\
\gamma_{z x b} \\
\gamma_{z x c} \\
\gamma_{z x 1} \\
\gamma_{z x 4} \\
\gamma_{z x 7}
\end{array}\right\}=\left[\begin{array}{cc}
\mathbf{I}_{3} & \mathbf{0}_{3 \times 3} \\
\mathbf{A} & \mathbf{B}
\end{array}\right]\left[\begin{array}{c}
\gamma_{z x a} \\
\gamma_{z x b} \\
\gamma_{z x c} \\
\tilde{\gamma}_{z x d} \\
\tilde{\gamma}_{z x e} \\
\tilde{\gamma}_{z x f}
\end{array}\right\} \text { or }\left\{\begin{array}{l}
\tilde{\gamma}_{z x d} \\
\tilde{\gamma}_{z x e} \\
\tilde{\gamma}_{z x f}
\end{array}\right\}=\mathbf{B}^{-1}\left\{\begin{array}{l}
\gamma_{z x 1} \\
\gamma_{z x 4} \\
\gamma_{z x 7}
\end{array}\right\}-\mathbf{B}^{-1} \mathbf{A}\left\{\begin{array}{l}
\gamma_{z x a} \\
\gamma_{z x b} \\
\gamma_{z x c}
\end{array}\right\}
$$

where

$$
\begin{aligned}
\mathbf{A} & =\left[\begin{array}{ccc}
-c_{1} & c_{2} & -c_{1} \\
-c_{1} & -c_{1} & c_{2} \\
c_{2} & -c_{1} & -c_{1}
\end{array}\right], \mathbf{B}=\left[\begin{array}{ccc}
c_{3} & c_{4} & c_{4} \\
c_{4} & c_{3} & c_{4} \\
c_{4} & c_{4} & c_{3}
\end{array}\right], c_{1}=0.6385595874119381 \\
c_{2} & =0.1263407264883946, c_{3}=1.8736592735116080, c_{4}=0.1385595874119366
\end{aligned}
$$

Similarly, we have

$$
\left\{\begin{array}{l}
\tilde{\gamma}_{z y d} \\
\tilde{\gamma}_{z y e} \\
\tilde{\gamma}_{z y f}
\end{array}\right\}=\mathbf{B}^{-1}\left\{\begin{array}{c}
\gamma_{z y 1} \\
\gamma_{z y 4} \\
\gamma_{z y 7}
\end{array}\right\}-\mathbf{B}^{-1} \mathbf{A}\left\{\begin{array}{c}
\gamma_{z y a} \\
\gamma_{z y b} \\
\gamma_{z y c}
\end{array}\right\}
$$

The above equations give the interpolated shear strains at the integration stations in terms of the sampled shear strains of AST10C.

Despite of the fact that $C R$ of AST10C is equal to $3 / 2$, AST10C does not appear to be more accurate than DISP10 in view of a number of popular benchmark problems !

\section{REFINED TRANSVERSE SHEAR STRAINS}

The primal variables in the conventional finite element method are displacement/rotations. To obtain strains, differentiation is used and renders strains one order less accurate than displacement/rotations except for a few optimal points [11]. In AST10C, some of shear strains are sampled at the element corners where the derivatives of the primal variables are often least accurate. These corner strains are deemed to be causes for the inferior accuracy of AST10C. To 
rectify the problem, the optimal edge strain points in a subparametric triangle are first identified. As the triangle can exactly interpolate a third order field, a fourth order field, say $f$, is now considered :

$$
f(x, y)=c_{1} x^{4}+c_{2} x^{3} y+c_{3} x^{2} y^{2}+c_{4} x y^{3}+c_{5} y^{4}
$$

The values of $f$ at the nodes of a subparametric triangle are evaluated and an interpolated $f$ can be obtained :

$$
\tilde{f}=\sum_{i=1}^{10} N_{i} f\left(x_{i}, y_{i}\right)
$$

After some straight forward but tedious mathematics, it is noted that :

- along the edge where $t=0,\left.\frac{d \tilde{f}}{d s}\right|_{s=(1-\eta) / 2},\left.\frac{d \tilde{f}}{d s}\right|_{s=1 / 2}$ and $\left.\frac{d \tilde{f}}{d s}\right|_{s=(1+\eta) / 2}$ agree with that of $\frac{d f}{d s}$ - along the edge where $r=0,\left.\frac{d \tilde{f}}{d t}\right|_{t=(1-\eta) / 2},\left.\frac{d \tilde{f}}{d t}\right|_{t=1 / 2}$ and $\left.\frac{d \tilde{f}}{d t}\right|_{t=(1+\eta) / 2}$ agree with that of $\frac{d f}{d t}$ - along the edge where $s=0,\left.\frac{d \tilde{f}}{d r}\right|_{r=(1-\eta) / 2},\left.\frac{d \tilde{f}}{d r}\right|_{r=1 / 2}$ and $\left.\frac{d \tilde{f}}{d r}\right|_{r=(1+\eta) / 2}$ agree with that of $\frac{d f}{d r}$ in which $\eta=\sqrt{5} / 3$. Hence, nine optimal edge strain points are identified. For a generic element, the natural transverse shear strains along the element edges are :

$$
\begin{aligned}
& \gamma_{z s}(s)=w(s, 1,2,3,4),{ }_{s}-y(s, 1,2,3,4),{ }_{s} \theta_{x}(s, 1,2,3,4)+x(s, 1,2,3,4),{ }_{s} \theta_{y}(s, 1,2,3,4) \quad \text { along } t=0 \\
& \gamma_{z t}(t)=w(t, 4,5,6,7),{ }_{t}-y(t, 4,5,6,7),{ }_{t} \theta_{x}(t, 4,5,6,7)+x(t, 4,5,6,7),{ }_{t} \theta_{y}(t, 4,5,6,7) \quad \text { along } r=0 \\
& \gamma_{z r}(r)=w(r, 7,8,9,1),{ }_{r}-y(r, 7,8,9,1),{ }_{r} \theta_{x}(r, 7,8,9,1)+x(r, 7,8,9,1),{ }_{r} \theta_{y}(r, 7,8,9,1) \text { along } s=0
\end{aligned}
$$

where

$$
\begin{aligned}
& w(\xi, i, j, k, l)=F_{1}(\xi) w_{i}+F_{2}(\xi) w_{j}+F_{3}(\xi) w_{k}+F_{4}(\xi) w_{l} \\
& F_{1}(\xi)=(2-3 \xi)(1-3 \xi)(1-\xi) / 2, F_{2}(\xi)=9 \xi(1-\xi)(2-3 \xi) / 2 \\
& F_{3}(\xi)=9 \xi(1-\xi)(3 \xi-1) / 2, F_{4}(\xi)=\xi(3 \xi-1)(3 \xi-2) / 2
\end{aligned}
$$


and interpolation relations similar to that of $w(\xi, i, j, k, l)$ hold for $x(\xi, i, j, k, l), y(\xi, i, j, k, l), \theta_{\mathrm{x}}(\xi, i, j, k, l)$ and $\theta_{\mathrm{y}}(\xi, i, j, k, l)$. In order to extend the optimal strains to the element corner, a quadratic interpolation is employed for the three optimal natural strains along each of the element edge, i.e.

$$
\begin{aligned}
& \tilde{\gamma}_{z s}(s)=G_{1}(s) \gamma_{z s}\left(s=\frac{1-\eta}{2}\right)+G_{2}(s) \gamma_{z s}\left(s=\frac{1}{2}\right)+G_{3}(s) \gamma_{z s}\left(s=\frac{1+\eta}{2}\right) \\
& \tilde{\gamma}_{z t}(t)=G_{1}(t) \gamma_{z t}\left(t=\frac{1-\eta}{2}\right)+G_{2}(t) \gamma_{z t}\left(t=\frac{1}{2}\right)+G_{3}(t) \gamma_{z t}\left(t=\frac{1+\eta}{2}\right) \\
& \tilde{\gamma}_{z r}(r)=G_{1}(r) \gamma_{z r}\left(r=\frac{1-\eta}{2}\right)+G_{2}(r) \gamma_{z r}\left(r=\frac{1}{2}\right)+G_{3}(r) \gamma_{z r}\left(r=\frac{1+\eta}{2}\right)
\end{aligned}
$$

where

$$
G_{1}(\xi)=\frac{(2 \xi-1)(2 \xi-1-\eta)}{2 \eta^{2}}, G_{2}(\xi)=\frac{-(2 \xi-1+\eta)(2 \xi-1-\eta)}{\eta^{2}}, G_{3}(\xi)=\frac{(2 \xi-1)(2 \xi-1+\eta)}{2 \eta^{2}}
$$

Thus, the interpolated optimal natural strains are $\tilde{\gamma}_{z r}(r=1)$ and $\tilde{\gamma}_{z s}(s=0)$ at node $1 ; \tilde{\gamma}_{z s}(s=1)$ and $\tilde{\gamma}_{z t}(t=0)$ at node 4 and $\tilde{\gamma}_{z t}(t=1)$ and $\tilde{\gamma}_{z r}(r=0)$ at node 7 . The optimal Cartesian strains are then obtained by tensorial transformation [12] :

$$
\begin{aligned}
& \left\{\begin{array}{l}
\tilde{\gamma}_{z x 1} \\
\tilde{\gamma}_{z y 1}
\end{array}\right\}=\left[\begin{array}{ll}
\left.\frac{d x(r, 7,8,9,1)}{d r}\right|_{r=1} & \frac{d y(r, 7,8,9,1)}{d r} \\
\left.\frac{d x(s, 1,2,3,4)}{d s}\right|_{s=0} & \left.\frac{d y(s, 1,2,3,4)}{d s}\right|_{s=0}
\end{array}\right]^{-1}\left\{\begin{array}{l}
\tilde{\gamma}_{z r}(r=1) \\
\tilde{\gamma}_{z s}(s=0)
\end{array}\right\} \\
& \left\{\begin{array}{l}
\tilde{\gamma}_{z x 4} \\
\tilde{\gamma}_{z y 4}
\end{array}\right\}=\left[\begin{array}{ll}
\left.\frac{d x(s, 1,2,3,4)}{d s}\right|_{s=1} & \frac{d y(s, 1,2,3,4)}{d s} \\
\left.\frac{d x(t, 4,5,6,7)}{d t}\right|_{t=0} & \left.\frac{d y(t, 4,5,6,7)}{d t}\right|_{t=0}
\end{array}\right]^{-1}\left\{\begin{array}{l}
\tilde{\gamma}_{z s}(s=1) \\
\tilde{\gamma}_{z t}(t=0)
\end{array}\right\}
\end{aligned}
$$

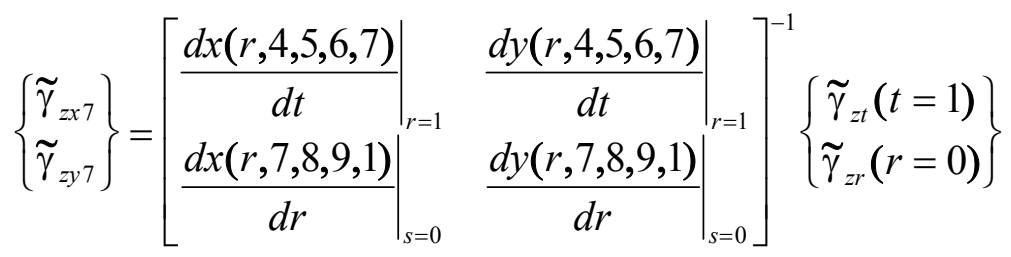

Using the interpolated strains in Eqn.(12) to replace $\gamma_{\mathrm{zx} 1}, \gamma_{\mathrm{zy} 1}, \gamma_{\mathrm{zx} 4}, \gamma_{\mathrm{zy} 4}, \gamma_{\mathrm{zx} 4}$ and $\gamma_{\mathrm{zy} 4}$ in Eqn.(8), a new set of strains at integration stations $d, e$ and $f$ is available. The so-formed element will be termed AST10O where "O” designates "optimal strains". 


\section{BENCHMARK PROBLEMS}

In this section, a number of popular plate bending benchmark tests are studied by the following element models :

DISP10 - the conventional displacement-based element.

AST10C - the assumed strain element using the displacement-derived strains at corner nodes.

AST10O - the assumed strain element using the interpolated optimal natural strains.

AST10I - same as AST10O except that the natural strains are sampled at the third order quadrature points of the element edges, i.e. $\eta=\sqrt{ }(3 / 5)$.

The rationale of including AST10I is that its nine sampled edge strains coincide with that of MITC12 [6-7]. Here, "I" designates "integration point".

Unless specified otherwise, all the finite element predictions will be normalized by their reference counterparts given in the textbook of Timoshenko \& Woinowsky-Krieger [13] and the material Poisson ratio is taken to be 0.3 .

Patch Test All the four elements pass the constant bending and transverse shear patch tests prescribed by MacNeal \& Harder [14].

Rank Examination The only zero energy modes of the four elements are the three rigid body modes, i.e. all the elements are rank sufficient.

Shear Locking Test The fully clamped $\left(w=\theta_{\mathrm{x}}=\theta_{\mathrm{y}}=0\right)$ square plate of side length $L$ and thickness $h$ is subjected to central point load. Owing to symmetry, only one quarter of structure is modelled, see Fig.4. The normalized central deflections are given in Table 1. Since $C R$ of all elements are larger than unity, none of the elements locks in this test. However, AST10I and AST10O yield the best results. AST10C is even less accurate than DISP10. The cause is deemed to be the practice employed by AST10C of sampling some shear strains at the element corner. Nevertheless, AST10C is less affected by the aspect ratio than DISP10 as noted in the predictions of the two elements $(0.595 / 0.672<0.364 / 0.399$ and $0.876 / 0.936<0.878 / 0.901)$.

Table 1. Normalized central deflections for the shear locking test, see Fig.4

\begin{tabular}{|ccccc|}
\hline Element & mesh & $L / h=100$ & $L / h=1,000$ & $L / h=100,000$ \\
\hline DISP10 & $1 \times 1$ & 0.672 & 0.595 & 0.594 \\
& $2 \times 2$ & 0.936 & 0.876 & 0.874 \\
\hline AST10C & $1 \times 1$ & 0.399 & 0.364 & 0.363 \\
& $2 \times 2$ & 0.901 & 0.878 & 0.878 \\
\hline
\end{tabular}




\begin{tabular}{|lllll|}
\hline AST10I & $1 \times 1$ & 0.990 & 0.979 & 0.979 \\
& $2 \times 2$ & 0.992 & 0.988 & 0.988 \\
\hline AST10O & $1 \times 1$ & 1.019 & 1.009 & 1.009 \\
& $2 \times 2$ & 0.996 & 0.992 & 0.992 \\
\hline
\end{tabular}

Square Plate Problems A quadrant of the square plate with $L / h=1,000$ is modelled by $2 \times 2,4 \times 4$ and $8 \times 8$ meshes. The plate is subjected to central point load and uniform pressure. Both simply supported $(w=0)$ and fully clamped $\left(w=\theta_{\mathrm{x}}=\theta_{\mathrm{y}}=0\right)$ conditions are attempted. The normalized central deflections and bending moments are listed in Tables $2 \mathrm{a}$ and $2 \mathrm{~b}$, respectively. AST10I and AST10O have the highest accuracy which is most obvious under the fully clamped condition.

Table 2a. Normalized central deflections for various square plate bending problems, see Fig.4

\begin{tabular}{|cccccc|}
\hline \multirow{2}{*}{ element } & & \multicolumn{2}{c}{ central point load } & \multicolumn{2}{c|}{ uniform pressure } \\
& mesh & simply supported & fully clamped & simply supported & fully clamped \\
\hline \multirow{3}{*}{ DISP10 } & $2 \times 2$ & 0.980 & 0.876 & 1.004 & 0.895 \\
& $3 \times 3$ & 0.996 & 0.956 & 1.005 & 0.971 \\
& $4 \times 4$ & 0.995 & 0.979 & 1.002 & 0.991 \\
\hline \multirow{3}{*}{ AST10C } & $2 \times 2$ & 0.980 & 0.878 & 1.010 & 0.943 \\
& $3 \times 3$ & 0.995 & 0.964 & 1.010 & 0.990 \\
& $4 \times 4$ & 0.998 & 0.983 & 1.007 & 0.999 \\
AST10I & $3 \times 2$ & 0.996 & 0.988 & 1.004 & 1.006 \\
& $3 \times 3$ & 1.002 & 0.996 & 1.007 & 1.004 \\
\hline \multirow{3}{*}{ AST10O } & $3 \times 4$ & 1.002 & 0.999 & 1.006 & 1.004 \\
& $3 \times 2$ & 0.997 & 0.992 & 1.004 & 1.006 \\
& $4 \times 4$ & 1.002 & 0.998 & 1.007 & 1.004 \\
\hline
\end{tabular}

Table 2b. Normalized central bending moments for square plates under uniform pressure, see Fig. 4

\begin{tabular}{|cccc|}
\hline element & mesh & simply supported & fully clamped \\
\hline \multirow{3}{*}{ DISP10 } & $2 \times 2$ & 1.143 & 1.107 \\
& $3 \times 3$ & 1.151 & 1.121 \\
& $4 \times 4$ & 1.033 & 1.067 \\
\hline \multirow{2}{*}{ AST10C } & $2 \times 2$ & 1.217 & 0.969 \\
& $3 \times 3$ & 1.120 & 1.028 \\
& $4 \times 4$ & 1.072 & 1.052 \\
\hline \multirow{2}{*}{ AST10I } & $2 \times 2$ & 1.019 & 0.963 \\
& $3 \times 3$ & 1.011 & 0.976 \\
& $4 \times 4$ & 0.999 & 0.986 \\
\hline \multirow{2}{*}{ AST10O } & $2 \times 2$ & 1.008 & 0.947 \\
& $3 \times 3$ & 1.007 & 0.971 \\
& $4 \times 4$ & 0.996 & 1.003 \\
\hline
\end{tabular}

Circular Plate Problems A circular plate of radius to thickness ratio equal to 500 is considered. A quadrant of the plate is modelled by 2, 6 and 24 elements, see Fig.5. The plate is subjected to central point load and uniform pressure. Both simply supported and fully clamped conditions are studied. The normalized central deflections and bending moments at the centre of the plate are 
listed in Tables 3a and 3b, respectively. Again, AST10I and AST10O yield the highest accuracy which is most obvious under the fully clamped condition.

Finally, the deflections along an axis of symmetry for the fully-clamped and centrally pointloaded plates with radius to thickness ratios equal to 500 and 5 are computed by using the mesh with six elements, see Fig.5. The results shown in Table 4 have been normalized by the thick plate solution given in reference [15]:

$$
w=\frac{P R^{2}}{16 \pi D}\left(1-\frac{r^{2}}{R^{2}}+\frac{2 r^{2}}{R^{2}} \ln \left(\frac{r}{R}\right)-\frac{8 D}{k G h r^{2}} \ln \left(\frac{r}{R}\right)\right)
$$

in which $P$ is the point load, $R$ is the radius of the plate, $D$ is flexural rigidity, $r$ is the radial coordinate and $G$ is the shear modulus.

Table 3a. Normalized central deflections for various circular plate problems, Fig.5

\begin{tabular}{|cccccc|}
\hline \multirow{2}{*}{ element } & $\begin{array}{c}\text { no. of } \\
\text { elements }\end{array}$ & \multicolumn{2}{c}{ central point load } & \multicolumn{2}{c|}{ uniform pressure } \\
& 2 & 0.999 & 0.038 & 1.019 & fully clamped \\
DISP10 & 6 & 0.993 & 0.857 & 1.023 & 0.135 \\
& 24 & 0.997 & 0.974 & 0.997 & 0.920 \\
\hline \multirow{3}{*}{ AST10C } & 2 & 0.813 & 0.907 & 0.833 & 1.095 \\
& 6 & 0.881 & 0.965 & 0.887 & 1.012 \\
& 24 & 0.995 & 0.991 & 0.996 & 1.002 \\
\hline \multirow{3}{*}{ AST10I } & 2 & 0.985 & 0.980 & 1.025 & 1.025 \\
& 6 & 0.996 & 0.988 & 1.024 & 1.003 \\
\hline \multirow{3}{*}{ AST10O } & 24 & 0.999 & 0.997 & 1.023 & 1.001 \\
& 2 & 0.989 & 0.989 & 1.022 & 1.018 \\
& 24 & 0.996 & 0.991 & 1.024 & 1.003 \\
\hline
\end{tabular}


Table 3b. Normalized central bending moments for circular plates under uniform pressure, Fig. 5

\begin{tabular}{|cccc|}
\hline element & no. of elements & simply supported & fully clamped \\
\hline \multirow{3}{*}{ DISP10 } & 2 & 0.956 & 1.310 \\
& 6 & 1.028 & 0.428 \\
& 24 & 1.000 & 0.916 \\
\hline \multirow{2}{*}{ AST10C } & 2 & 1.097 & 1.377 \\
& 6 & 0.967 & 1.094 \\
AST10I & 24 & 1.011 & 1.036 \\
& 2 & 0.896 & 0.750 \\
& 6 & 0.987 & 0.980 \\
AST10O & 24 & 0.997 & 0.991 \\
& 2 & 0.876 & 0.725 \\
& 6 & 0.984 & 0.983 \\
& 24 & 0.996 & 0.989 \\
\hline
\end{tabular}

Table 4. Normalized deflections for thin/thick circular plate under central point load, see Fig.5

\begin{tabular}{|c|c|cccccc|}
\hline \multicolumn{2}{|c|}{$r / R$} & $0 / 6$ & $1 / 6$ & $2 / 6$ & $3 / 6$ & $4 / 6$ & $5 / 6$ \\
\hline \multirow{3}{*}{$R / h=500$} & DISP10 & $0.857^{*}$ & 0.884 & 0.875 & 0.834 & 0.788 & 0.651 \\
& AST10C & $0.965^{*}$ & 0.999 & 1.000 & 1.022 & 1.045 & 1.073 \\
& AST10I & $0.988^{*}$ & 1.002 & 1.001 & 1.002 & 1.015 & 1.072 \\
& AST10O & $0.991^{*}$ & 1.002 & 1.000 & 1.002 & 1.014 & 1.069 \\
\hline \multirow{3}{*}{$R / h=5$} & DISP10 & $1.394^{*}$ & 1.003 & 0.990 & 1.005 & 1.004 & 0.980 \\
& AST10C & $1.372^{*}$ & 1.009 & 1.008 & 1.013 & 1.017 & 1.036 \\
& AST10I & $1.388^{*}$ & 1.009 & 0.999 & 1.008 & 1.008 & 0.987 \\
& AST10O & $1.391^{*}$ & 1.009 & 0.999 & 1.008 & 1.006 & 0.984 \\
\hline
\end{tabular}

* normalized by the thin plate solution, i.e. the last term in Eqn.(14) which tends to infinity as $r$ tends to zero is excluded

\section{CLOSURE}

A simple assumed strain method is devised to improve the accuracy of the ten-node triangular plate bending element by shifting three of the strain sampling points from integration stations to the element corners. To obtain the shear strains at the corners, the natural shear strains tangential to the element edges are interpolated. Two sets of interpolation pivots are adopted. The first set contains points which are optimal for the derivatives of a prescribed fourth order field. The second set contains the integration stations of the third order quadrature. Numerical tests indicates that both set of pivots yield elements of close accuracy which is consistently higher than that of the conventional element.

Acknowledgment - The financial support of Hong Kong Research Grant Council is gratefully acknowledged. The authors are indebted to Professor Theodore H. H. Pian for his valuable comments. 


\section{REFERENCES}

1. K.Y.Sze, D.Zhu, D.-P.Chen, "Quadratic triangular $\mathrm{C}^{0}$ plate bending element”, Inter.J.Numerical Methods Engrg., 40, 937-951 (1997)

2. K.Y.Sze, "On the relative merits of three-point integration rules for six-node triangles", Finite Elements in Analysis \& Design, accepted and in press

3. K.Y.Sze, D.Zhu, D.-P.Chen, "Formulation of six-node $\mathrm{C}^{0}$ curved triangular shell elements", Inter.J. Numerical Methods Engrg., submitted

4. K.Y.Sze, D.Zhu, "A quadratic assumed natural strain curved triangular shell element", Computer Methods Applied Math.Mech., submitted

5. S.W.Lee, C.C.Dai, C.H.Yeom, "New triangular finite element for thin plates and shells", Inter.J.Numer. Methods Engrg., 21, 1813-1831 (1985)

6. K.-J.Bathe, M.L.Bucalem, F.Brezzi, "Displacement and stress convergence of our MITC plate bending elements", Engineering Computation, 7, 291-302 (1990)

7. K.-J.Bathe, Finite Element Procedures, Prentice Hall, New Jersey, 1996

8. T.J.R.Hughes, The Finite Element Method - linear static and dynamic finite element analysis, Prentice-Hall, New Jersey, 1987

9. R.D.Cook, D.S.Malkus, M.E.Plesha, Concepts and Applications of Finite Element Analysis, $3^{\text {rd }}$ edn., John Wiley \& Sons, New York, 1989

10.O.C.Zienkiewicz, R.L.Taylor, The Finite Element Method, $4^{\text {th }}$ Edn., McGraw-Hill, London, 1989

11. J.Barlow, "More on optimal stress points - reduced integration, element distortions and error estimation", Inter.J.Numer.Methods Engrg., 28, 1487-1504 (1989)

12. Y.C.Fung, Foundation of Solid Mechanics, Prentice-Hall, Englewood Cliffs, New Jersey, 1965

13.S.P.Timoshenko, S.Woinowsky-Krieger, Theory of Plates and Shells, 2nd Edn., McGraw-Hill, New York, 1970

14. R.H.MacNeal, R.L.Harder, "A proposed standard set of problems to test finite element accuracy", Finite Elements in Analysis \& Design, 1, 3-20 (1985)

15.B.L.Wong, T.Belytschko, "Assumed strain stabilization procedure for the 9-node Lagrange shell elements”, Engrg.Comput., 4, 229-239 (1987) 


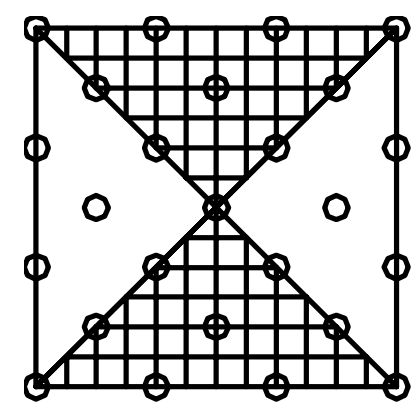

(a)

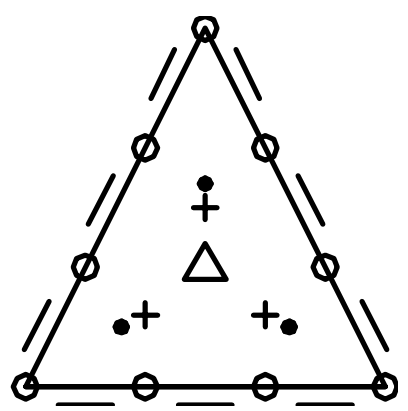

(b)

Fig.1 (a) A quadrilateral macro-element composed of two kinds of hybrid strain elements [5]

(b) MITC12 element with nine boundary nodes and four bubbles nodes ( $\mathbf{O}$ denotes a node

with full d.o.f.s, - denotes a node with only rotational d.o.f.s, $\triangle$ denotes a node with

only translational d.o.f.s, - denotes a sampled shear strain

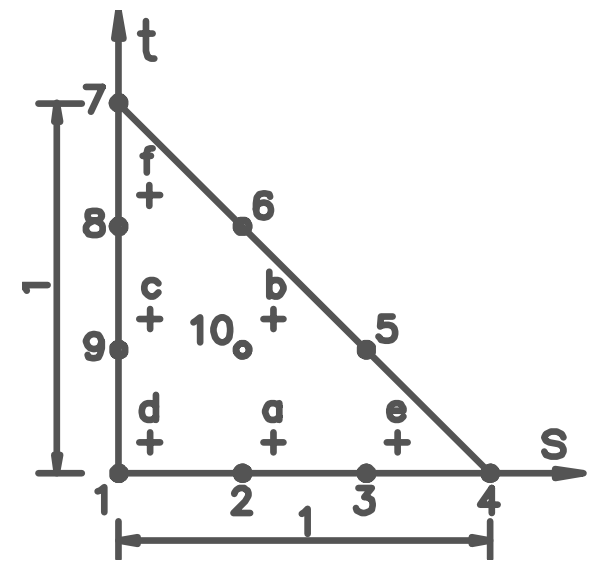

(a)

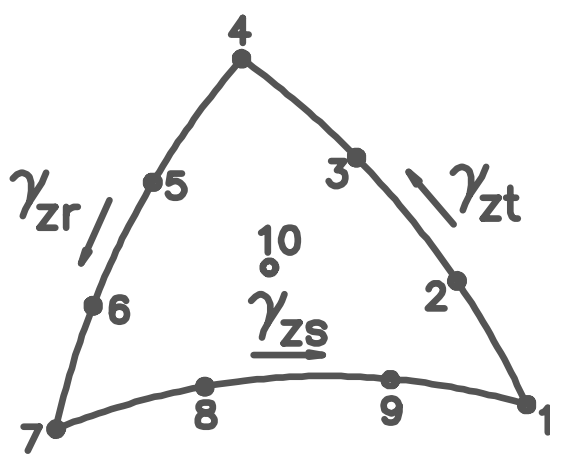

(b)

Fig.2 (a) The ten-node triangular element and the integration stations of the six-point rule

(b) The natual transverse shear strains along the element edges 


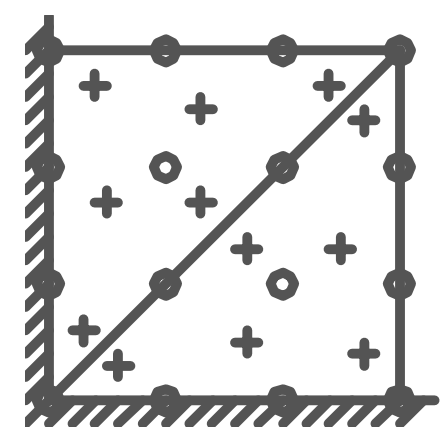

(a)

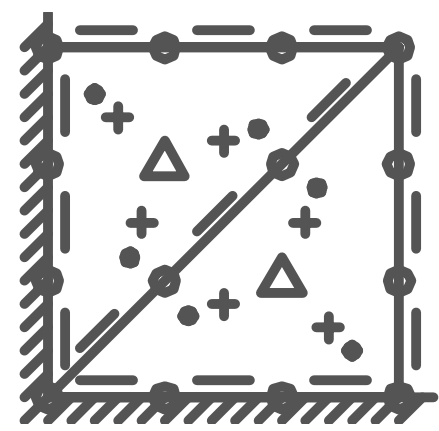

(b)

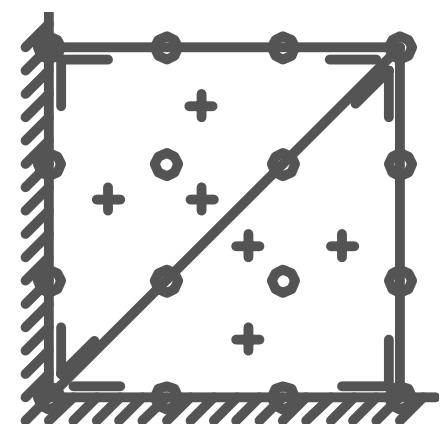

(c)

Fig.3 Constraint counts for (a) DISP10, (b) MITC12 and (c) AST10's

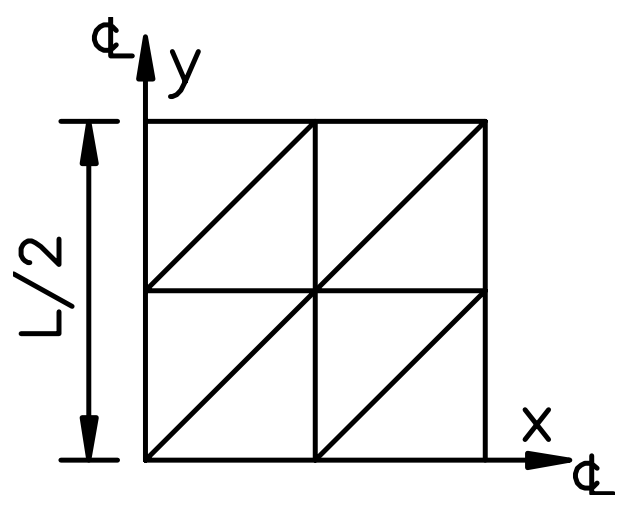

Fig.4 A quadrant of a square plate modelled by a $2 \times 2$ mesh
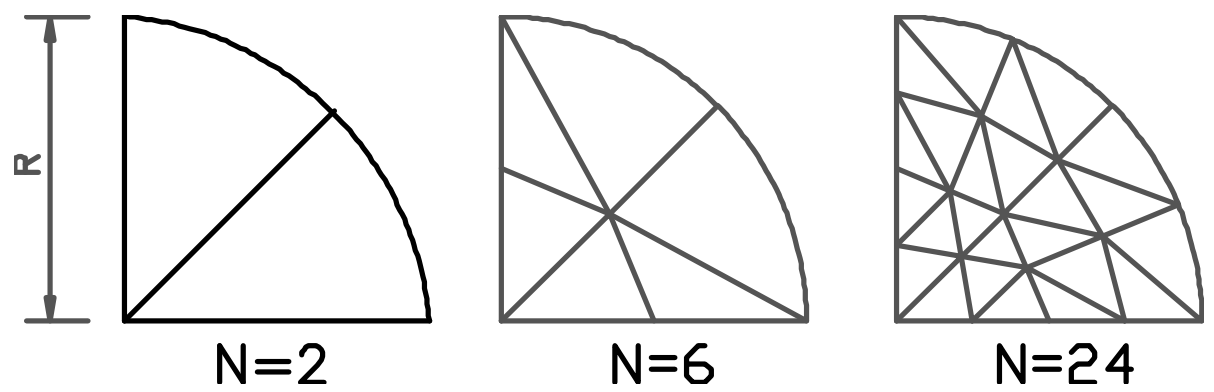

Fig.5 Meshes for a quadrant of circular plate ( $\mathrm{N}$ is the number of element) 